\title{
Mass transfer studies across ventilated hydrofoils: A step towards hydroturbine aeration
}

Ashish Karn $_{1,4,2}^{1,2}$, Garrett M. Monson ${ }^{3}$, Christopher R. Ellis ${ }^{1}$, Jiarong Hong ${ }^{1,2}$, Roger E.A. Arndt ${ }^{1,4}$, John S. Gulliver ${ }^{1,4}$,

1. Saint Anthony Falls Laboratory, 2 Third Ave. SE, University of Minnesota, Minneapolis, MN, USA.

2. Department of Mechanical Engineering, University of Minnesota, Minneapolis, MN, USA

3. Houston Engineering, Maple Grove, MN, USA.

4. Department of Civil Engineering, University of Minnesota, Minneapolis, MN, USA.

* Email addresses of the corresponding author: gulli003@umn.edu

\begin{abstract}
The water discharged by hydropower facilities is a matter of increasing concern due to poor downstream water quality. The use of auto-venting hydroturbines has been suggested as one of the best ways to mitigate low dissolved oxygen levels in the downstream water. Much of the design of auto-venting hydroturbines is currently performed with computational fluid dynamics (CFD) simulations. However, there is little information available to test and verify the performance of these simulations regarding gas transfer and bubble size distribution. This paper investigates the performance of a water tunnel test-bed for CFD simulations of an auto-venting hydroturbine through the use of a ventilated hydrofoil. Bubble size distributions are measured by a shadow imaging technique and found to have a Sauter mean diameter of $0.9 \mathrm{~mm}$ for a reference case. Higher liquid velocities, a lower airflow rate and a higher angle of attack all resulted in a greater number of small bubbles and a lower weighted mean bubble size. Bubble-water oxygen transfer is measured by the disturbed equilibrium technique. The gas transfer model of Azbel (1981) is utilized to characterize the liquid film coefficient for oxygen transfer, with one scaling coefficient to reflect the fact that characteristic turbulent velocity is replaced by cross-sectional mean velocity. The value of the coefficient is found to stay constant at a particular hydrofoil configuration while it varied over a narrow range of 0.52-0.60 for different hydrofoil angles of attack. This suggests that it is an appropriate coefficient for flow over a ventilated hydrofoil and possibly other flow situations. These results can be used by investigators to test and verify their CFD model against known bubble size distributions and gas transfer in a water tunnel flow that has important similarities to an auto-venting hydroturbine.
\end{abstract}

Keywords: Hydroturbine aeration, Gas transfer, Bubble-water transfer, Ventilated hydrofoil, Auto venting turbines, Mass transfer model, Liquid film coefficient, Bubble size distribution, Shadow imaging technique. 


\section{Introduction and Motivation}

Due to the depletion of fossil fuel supplies, global climatic changes and concern over nuclear waste management, the electric power industry is focusing attention on renewable energy technologies such as hydropower. Conventional hydropower has the potential to contribute a substantial portion of our energy requirements. However, the water discharged by hydropower facilities is of increasing concern due to its effect on downstream water quality [1,2]. This decreased water quality arises from two different mechanisms: increased dissolved gases such as nitrogen over high spillways and greatly diminished oxygen content in the water discharged from hydroturbines to the downstream environment.

The impoundments necessary for creating the hydraulic head to operate conventional hydroturbines can degrade water quality. The residence time of water within these reservoirs is long and processes such as respiration by aquatic plant and animal life, biodegradation of organic materials in the sediments, oxygen-consuming chemical reactions, etc. can decrease the DO levels, especially at greater depths (i.e. the hypolimnion) within the reservoir. Thermal stratification due to solar heating enhances conditions for low DO in the hypolimnion. Such a system, being hydrodynamically stable, inhibits mixing between layers and isolates the bottom water from atmospheric oxygen. Only surface waters are replenished with oxygen through gas transfer processes resulting from wave action [3]. Hydropower projects often have hydroturbine intakes located in the hypolimnion where DO levels may drop to anoxic conditions $[4,5]$. Hypolimnetic anoxia in turn leads to trace metals, nutrients, and hydrogen sulfide being released from sediments and a drop in the $\mathrm{pH}$ of the water endangering fish and other aquatic life in downstream rivers [6]. 
Often, air injection is suggested as a method to improve DO concentration in lakes and rivers [710]. Research on oxygen mass transfer between air and water has focused on free surface flows viz. gas transfer in spillway discharges [11-13]. The auto venting turbine (AVT) has been proposed as a cost-effective and promising technology that can be employed to mitigate the problems associated with low DO concentration in the hydroelectric releases $[14,15]$. The AVT is a self-aspirating hydroturbine designed to aerate the turbine discharge through ports located at low pressure regions which are connected to the atmosphere. Air released to the water from these ports breaks up into small bubbles due to the water's high velocity and turbulence. Oxygen transfer is augmented by the high interfacial area of these bubbles [16]. Relatively limited research has been undertaken on optimizing the performance of auto venting systems.

There are three important factors influencing the performance of an AVT: the quantity of entrained air, the bubble sizes and the rate of oxygen transfer from the bubbles. Further, computational models to study the impact of entrained air on the flow field and vice versa need to be developed. Such computational models need to quantify DO transfer as a function of flow conditions occurring in AVTs. To validate such a computational model, a dataset that allows quantitative analysis of aeration statistics for different hydroturbine blade hydrofoil designs is required. Such a dataset must provide information on bubble sizes, void fraction in the wake and the rate of oxygen transfer in the wake as a function of the different flow parameters. An aeration test-bed should be capable of testing these computational codes using configurations similar to an AVT. Such an aeration test-bed will advance the development and implementation of aerating hydroturbines at hydropower facilities, reduce the cost and regulatory uncertainty prior to hydropower development and enhance the aeration design capabilities. 
In this study, a series of air injection experiments were conducted on a hydrofoil at several hydrodynamic conditions to investigate the effects of flow field on the size of the bubbles generated and the resulting rate of oxygen transfer. Experiments conducted to establish the methodology for creating a test bed were designed to study the impact of varying water velocities, airflow, and hydrofoil orientation (angle of attack) on the bubble sizes generated, void fraction in the wake, and oxygen transfer from the bubbles. A one-dimensional mass transfer model is developed to lend insight into the mass transfer characteristics observed in these experiments.

\section{Description of experimental setup and methodology}

\subsection{Experimental apparatus}

The experiments are conducted in the Saint Anthony Falls Laboratory (SAFL) high-speed water tunnel at the University of Minnesota [17, 18]. As shown in Figure 1, the tunnel has a horizontal test section of $1 \mathrm{~m}$ (length) $\times 0.19 \mathrm{~m}$ (width) $\times 0.19 \mathrm{~m}$ (height) with three side walls made of plexiglass for optical access. The tunnel is designed for cavitation and air ventilation studies and is capable of velocities in excess of $20 \mathrm{~m} / \mathrm{s}$. The gas collector dome (in the settling chamber) of the tunnel provides for the removal of large quantities of air injected as part of cavitation and ventilation experiments, thus allowing such experiments to continue for extended periods of time with little effect on test section conditions.

Validyne AP-10 absolute pressure transducers are used to measure absolute pressure in the test section and in the elbow vane downstream of the diffuser. A Validyne DP-10 differential pressure transducer is employed to measure the differential pressure between the settling chamber and test section and used to calculate test section velocity. The pressure transducers are calibrated before each series of experiments. The calibrations are performed using mercury 
manometer for both transducers.. The pressure transducer calibrations are linear, with R-squared values typically 0.9999 or higher for both transducers. Standard errors of the pressure calibrations are approximately $0.1 \mathrm{kPa}$ for both absolute pressure transducers. These errors lead to a maximum error in the measured velocity of $0.11 \mathrm{~m} / \mathrm{s}$, with typical errors being closer to 0.02 $\mathrm{m} / \mathrm{s}[19]$.

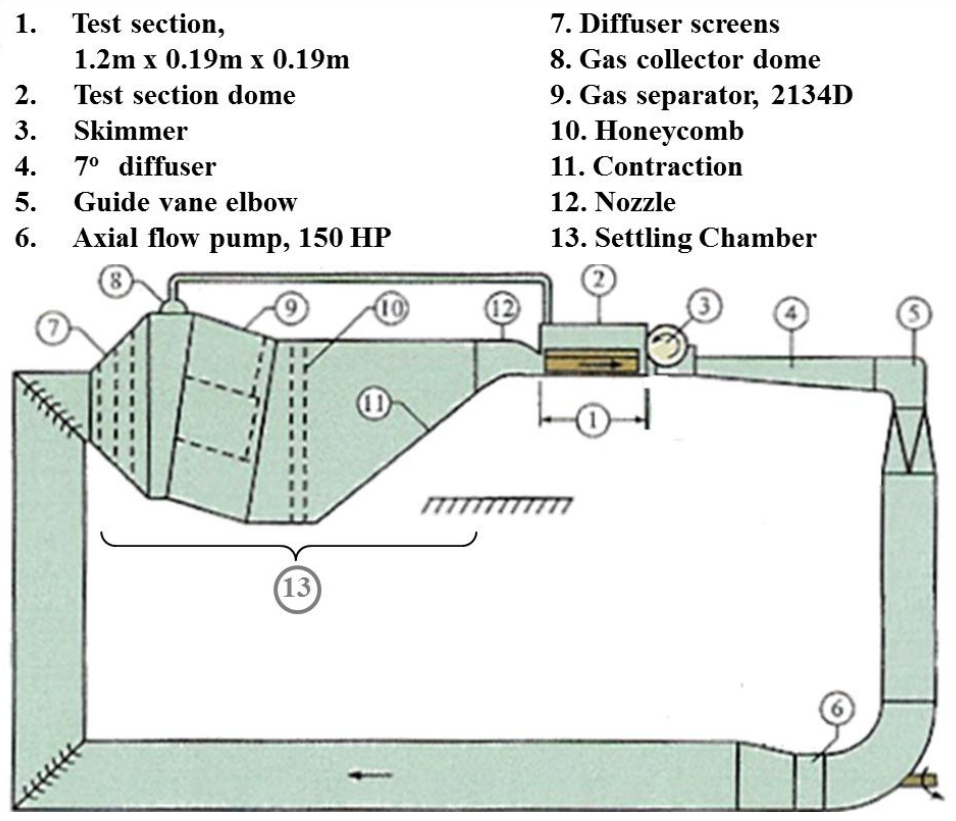

Figure 1: Schematic of the SAFL water tunnel facility.

A YSI thermistor probe is used to monitor water temperature in the water tunnel. An Omega Engineering FMA-2609A mass flow controller is used to set and measure airflow to the hydrofoil. Two Hach luminescent dissolved oxygen (LDO) probes and controller are used to measure DO concentration in both the settling chamber and the elbow vane downstream of the diffuser.

\subsection{Hydrofoil and the flow conditions}

During the experiments, a 2-D NACA0015 hydrofoil is installed horizontally in the test section and adjusted to one of three different angles of attack $(\alpha): 0^{\circ}, 4^{\circ}$ and $8^{\circ}$. The hydrofoil is $190 \mathrm{~mm}$ 
in span and $81 \mathrm{~mm}$ in chord. As shown in Figure 2, a narrow spanwise slot allowed air to be injected into the flow near the leading edge of the hydrofoil on its suction side. For the gas transfer measurements, the full span length of the injection slot is used for air injection. This results in a dense spanwise bubbly wake. However, in order to make bubble measurements, ventilation is limited to a narrow $9.6 \mathrm{~mm}$ long slot section (5\% of the full slot length) at the center of the span. This configuration ensures that bubbles remain within a narrow depth of field from the center of the test section. Videos of the bubbly wake verify that the lateral drift or spread of the bubbles is minimal. Silberman [20] investigated the production of bubbles by an orifice in liquid shear flows. His findings indicated that the bubble diameter depends only on air injection rate and water velocity, not on orifice size, as long as the gas issues as a jet from the orifice. Similarly, it is believed that the bubble sizes coming out of the injection slot are not dependent on the slot width. Since the injection slot width is constant in our experiments, it is reasonable to posit that the bubbles produced from the limited (5\%) slot length are representative of the bubble population that exists when the full span is ventilated. For these bubble size analysis experiments, air flow rates were selected that were $5 \%$ of those used in the gas transfer testing. Thus, for any air flow test condition, the air flow rate per unit slot length was kept constant. Fifteen different experiments were conducted at test section water speeds of 5, 7.5 and $10 \mathrm{~m} / \mathrm{s}$, ventilation gas flow of 10, 20 and 30 LPM per unit span and hydrofoil angle of attack (AoA) of 0, 4 and 8 degrees. The bubbly wake images are obtained at 3 streamwise distances of 109, 243, and $377 \mathrm{~mm}$ downstream of the hydrofoil center (or 1.3, 3.0 and 4.7 times the chord length, respectively.) The resulting bubble diameters were close to the same, and the $377 \mathrm{~mm}$ location was chosen because it was believed to have the best view of the bubbles. 

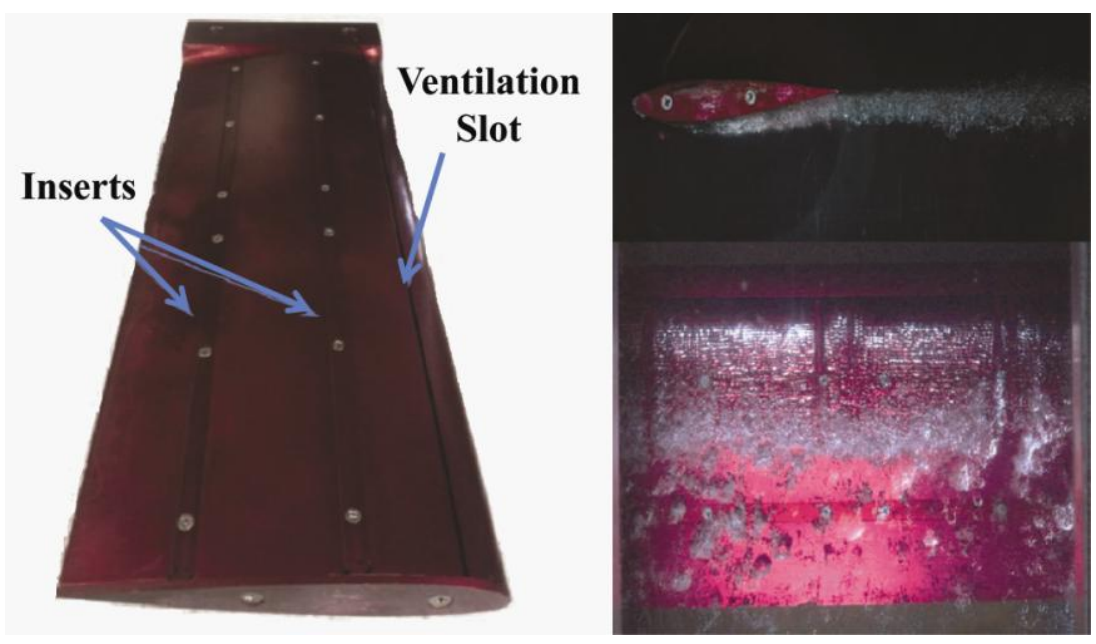

Figure 2: Details of the NACA0015 ventilated foil. Left: photo of suction side of hydrofoil. Right: Sample image of ventilated flow. Side view and bottom view of suction side.

\subsection{Experiment Methodology: Degassing and Imaging.}

A disturbed equilibrium technique is used to estimate gas transfer rates by initially degassing the water to the lowest level possible and then reoxygenating the water while taking continuous DO concentration measurements [21]. During deoxygenation, a portion of water is withdrawn from the tunnel and circulated through a degassing chamber under vacuum where the water is discharged into the chamber through nozzles, creating a cloud of falling droplets. The degassing loop is run overnight, reducing the DO concentration to approximately $4 \mathrm{ppm}$. Immediately prior to an aeration test, the water tunnel is run without air injection until the water is thoroughly mixed. A test commences by setting the test section velocity and airflow rate. The experiment is run for approximately 10 hours [19] or until the oxygen concentration exceeds $99 \%$ of saturation. A shadow imaging technique $[22,23]$ with direct, in-line volume illumination using a pulsed LED array source is used to capture shadow images of the bubbly wake similar to the study of Karn et al. [24]. Figure 3 shows a schematic of the experimental setup. A custom-made pulsed LED light source from Innovative Scientific Solutions Inc. is used to illuminate the flow. The pulsed LED array has flash rates up to $10 \mathrm{kHz}$ with a $5 \mu$ s minimum pulse width and rise and fall 
times of $200 \mathrm{~ns}$. To improve the uniformity of the back-lighting in the images, a light shaping diffuser is placed between the light source and the flow. A $1 \mathrm{~K} \times 1 \mathrm{~K}$ pixel Photron APX-RS camera, capable of 3000 frames/second at full resolution is used to obtain images. To minimize blur, all exposure times are $15 \mu$ s and the frame rate is 25 images per second. The focus plane is located at the center of the test-section and the depth of field is determined to be approximate 10 $\mathrm{mm}$. The captured images have a spatial resolution of $0.059 \mathrm{~mm} /$ pixel and the bubble sizes are in the range of 1 to 68 pixels, which corresponds to a bubble diameter of 0.12 to $4 \mathrm{~mm}$. A data set for a single experiment consists of 1000 images taken over a 40 second period.

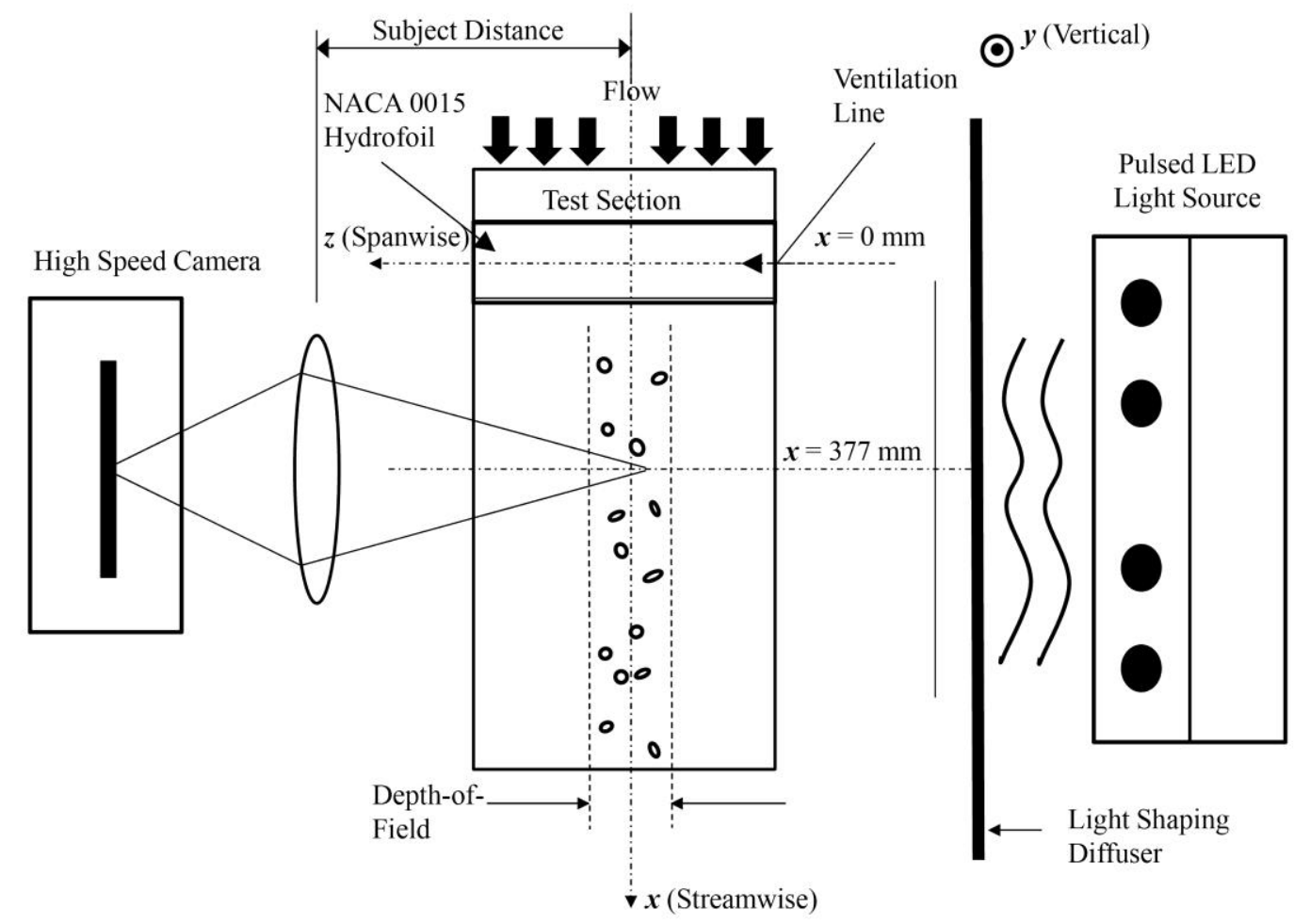

Figure 3: Schematic of the experimental setup for shadow imaging of bubbly flows in an aerated hydrofoil wake adapted from Karn et al. [24] 


\subsection{Image processing and analysis}

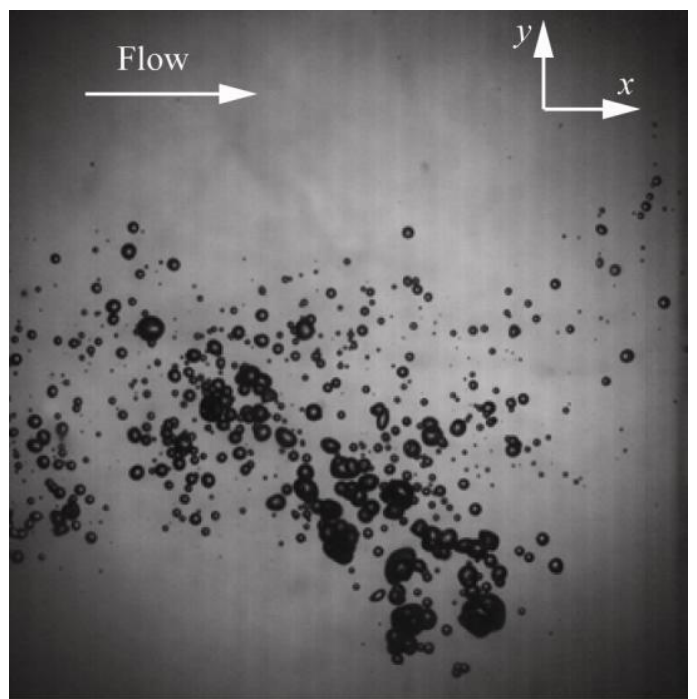

Figure 4: A sample bubbly wake image obtained in shadow imaging experiments.

Figure 4 provides a sample bubble image from our experiments. The bubbly wake is characterized by a wide bubble size distribution ranging from microbubbles to large bubble clusters. The bubbles could be either in-focus or slightly out-of-focus. An image analysis technique, as described in Karn et al. [24], is employed to extract the information about the bubble locations and the bubble sizes from the bubble images. It consists of three major steps. First (1), the original grayscale images are binarized. This is followed by (2) categorization of bubble regions. The bubble regions are labeled and characterized by a series of metrics including area, centroid, major and minor radius (projected bubbles are assumed to be ellipses) and a circularity factor. Based on the area of each region, the bubbles are divided into tiny spherical bubbles, intermediate-sized bubbles and large bubbles or clusters. Lastly (3), bubble separation and information extraction is performed. A multilevel segmentation approach is used to extract the bubble locations and dimensions from the images. 'Cluster Processing' using advanced morphological operations and a "watershed transform" [25] extracts individual bubbles from 
bubble clusters. The information for all identified bubbles including their $x$ and $y$-coordinates and minor and major radius is then tabulated [24].

\section{Mass Transfer Model}

A one-dimensional model of the change in dissolved oxygen (DO) concentration in the tunnel was developed to fit the results of the physical experiments to a mass transfer theory using a single coefficient. The simplified conservation equation for the concentration of oxygen in a section of the tunnel is given by equation 1 as:

$U \frac{d C}{d x^{+}}=k_{L} a\left(C_{s}-C\right)$

where $C$ refers to DO concentration and $C_{S}$ corresponds to equilibrium DO concentration in a particular section of the tunnel, $x^{*}$ refers to a moving co-ordinate system given as $x^{*}=x-U t, U$ is cross-sectional mean velocity, $k_{L}$ is the liquid film coefficient for transfer of a volatile gas across the bubble-liquid interface, and $a$ is specific surface area of bubbles (or the surface area of bubbles divided by volume associated with those bubbles), given by equation 2 below:

$a=\frac{\Lambda_{\mathrm{St}}}{V_{\mathrm{w}}}$

Where $A_{\mathrm{St}}$ is the total surface area of the bubbles and $V_{\mathrm{w}}$ is the volume of water in which $A_{S t}$ is determined. Bubble diameter is determined from from the shadow imaging experiments, with limited width, and surface area weighted bubble diameter is defined as:

$d_{2}=\sqrt{\frac{\Lambda_{s i}}{\pi N_{s i}}}$

where $A_{s i}$ and $N_{s i}$ are the bubble surface area and the number of bubbles from the shadow imaging experiments, respectively. Next, it is assumed that the limited width of the shadow imaging experiments does not alter this ratio: 
$\frac{\Lambda_{s i}}{N_{s i}}=\frac{\Lambda_{s t}}{N_{t}}$

where $N_{\mathrm{t}}$ is the total number of bubbles corresponding to $A_{S t}$. Then, the combination of equation 3 and 4 gives:

$A_{s \mathrm{t}}=\pi d_{2}^{2} N_{\mathrm{t}}$

The void fraction of air $(\varphi)$ is given as:

$\varphi=\frac{V_{b}}{V_{w}}=\frac{N_{t} \pi d_{3}^{2}}{6 V_{w}}$

where $V_{b}$ is the total volume of bubbles in control volume $V_{w}$, and $d_{3}$ is the volume weighted bubble diameter determined from the shadow imaging experiments:

$d_{3}=\left(\frac{6 V_{s i}}{\pi N_{s i}}\right)^{1 / 3}$

where $V_{s i}$ is the total volume of bubbles from the shadow imaging experiments, and equation 6 may be solved for $N_{t}$ :

$N_{t}=\frac{6 \varphi V_{w}}{\pi d_{3}^{3}}$

Equations 2, 5 and 8 may be combined to give:

$a=6 \varphi \frac{d_{2}^{2}}{d_{3}^{3}}=\frac{6 \varphi}{d_{32}}$

where $d_{32}$ is the Sauter mean diameter of the generated bubble population [26].

The equilbrium concentration $C_{\%}$ is given by Henry's law and varies with temperature, pressure and solute content $[13,27]$. The mass transfer coefficient $k_{L}$ depends on the local air void fraction, the geometrical characteristics of the bubbles and local flow characteristics. Therefore, 
$k_{L}$ may be approximated by predictive relations $[16,21,28]$, but is best determined by controlled, flow-specific laboratory experiments. The theory of Azbel [28] is employed to determine the liquid film coefficient in the model, $k_{\mathrm{L}}$ as:

$k_{\mathrm{L}}=\beta \frac{D^{1 / 2}}{4 \pi} \frac{u^{3 / 4}}{\left(v_{f} l\right)^{1 / 4}} \frac{(1-\varphi)^{1 / 2}}{\left(1-\varphi^{5 / 3}\right)^{1 / 4}}$

Where, $D$ is the diffusion coefficient of the solute (oxygen) in water, $v_{f}$ is kinematic viscosity, $l$ is a characteristic turbulent length scale, $u$ is a characteristic turbulent velocity, and $\beta$ is a coefficient on the order of 1 to be determined by fitting with the experimental data.

The one-dimensional mass transfer model requires certain inputs such as water velocity, testsection pressure and temperature and the initial and steady state oxygen concentrations. The model partitions the water tunnel into six sections: test section, diffuser, right leg, bottom leg, left leg, and settling chamber. It requires inputs of test section velocity, test section pressure, average bubble diameter, airflow rate, water temperature, initial DO concentration, and DO system steady state or saturation concentration (referred to as 'saturation concentration' from now on). A section's cross-sectional mean velocity is substituted for characteristic turbulent velocity, the hydraulic radius of each section is substituted for turbulent length scale and the total bubble surface area is adjusted based on the change in bubble diameter caused by mass transfer and pressure changes using the ideal gas law. The model begins in the test section where it calculates $k_{\mathrm{L}}$ based on the inputs and tunnel geometry and an assumed value of $\beta$. A fourth-order Runge Kutta method is employed to solve for the change in DO that results from the water's transit of the test section. The model then steps to the next section of the tunnel where it executes the same procedure using the DO concentration that leaves the previous tunnel section as the section's inlet concentration. This process is repeated for all remaining tunnel sections. The bubbles then leave 
the tunnel via the gas collector dome above the low velocity settling chamber. The loop is then repeated with the new concentration in the settling chamber as the inlet concentration to the test section, where bubbles are injected into flow at the foil. This process is repeated until a steady

state concentration is reached. The time it takes for the water to complete one loop is known based on calculated cross-section mean velocities throughout the water tunnel. This time is used to compare the calculated to measured DO concentrations where the LDO probes are located as a function of time.

A nonlinear regression was then undertaken on the coefficient $\beta$. An initial value of $\beta$ was chosen, and varied by the slope-intercept method until the optimum curve-fit was achieved, as indicated by the minimum residual sum of squares.

\section{Results and Discussion}

\subsection{Results on Bubble Size}

A reference case, chosen to introduce the reader to a bubbly wake of a ventilated hydrofoil, will be presented herein. The reference case is at zero degrees AoA, a water velocity of $5 \mathrm{~m} / \mathrm{s}$ and a ventilation mass airflow rate of 10 SLPM (standard liters per minute). Figure 5 shows the probability density functions (PDF) of number of bubbles, total surface area and total volume as functions of bubble diameter in the wake of the foil The PDF of bubble number illustrates the large fraction of very small size bubbles in the wake. As can be seen from the plot, more than $40 \%$ of the bubbles are around $0.3 \mathrm{~mm}$ in diameter. Liao and Lucas [30] suggest that these smallsized bubbles are not generated at the air injection point but are produced as a result of breakup processes related to increased turbulence in the wake. 


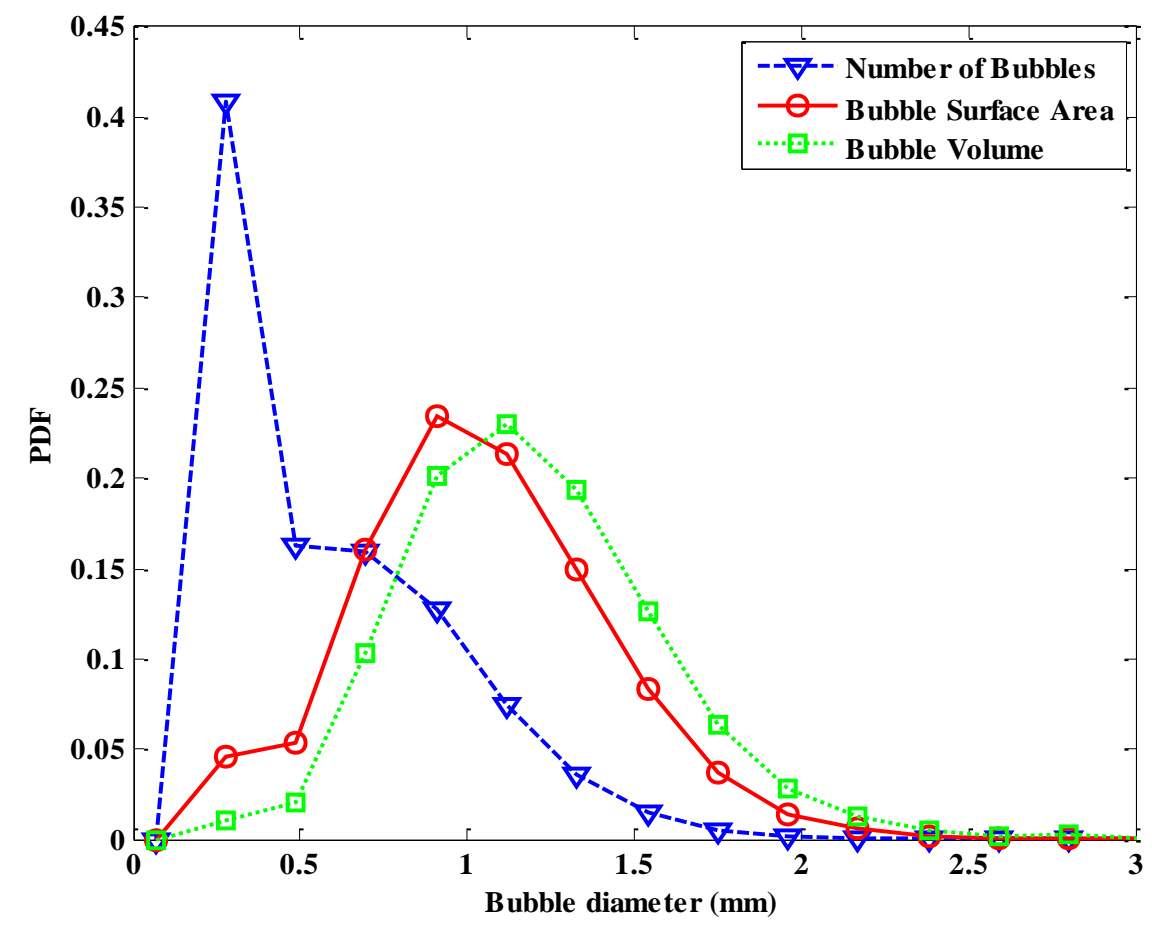

Figure 5: PDFs of number of bubbles, bubble surface area and volume in the bubbly wake for the reference case.

The PDFs of the bubble surface area and bubble volume exhibit maxima just below and just above a bubble diameter of $1 \mathrm{~mm}$, respectively. Around $24 \%$ of the entire surface area of the bubbles is contributed by bubbles with a diameter of between $0.8 \mathrm{~mm}$ and $1 \mathrm{~mm}$. An equal amount of total bubble surface area is represented by slightly larger bubbles with diameters between $1 \mathrm{~mm}$ and $1.2 \mathrm{~mm}$. It should be noted that the bubble size associated with the mean value of total surface area or volume denotes Sauter mean diameter (denoted interchangeably by SMD or $d_{32}$ ) or volume mean diameter (VMD or $d_{43}$ ), respectively. From the figure above, the approximate value of SMD and VMD are seen to be $0.95 \mathrm{~mm}$ and $1.1 \mathrm{~mm}$ respectively.

In contrast, mean surface diameter (i.e. MSD or $d_{20}$ ) and mean volume diameter (i.e. MVD or $d_{30}$ ) represent a bubble size that if all the bubbles were of this diameter, then the total surface area and volume of the bubbles, respectively, would be the same as in the actual bubble population. 
In general, for a distribution of bubbles of similar shape, a weighted mean diameter $d_{\mathrm{mn}}$ is defined as:

$d_{m \pi}=\left[\frac{\sum n_{i} d_{i}^{m}}{\sum n_{i} d_{i}^{n}}\right]^{1 /(n-n)}$

where the summation is carried out over the variable $i$. The table below shows the variation of these different representative diameters for the bubble size distribution for the reference case:

\begin{tabular}{|c|c|c|c|}
\hline Nomenclature & $\boldsymbol{m}$ & $\boldsymbol{n}$ & $\boldsymbol{d}_{\boldsymbol{m n}}(\mathbf{m m})$ \\
\hline Mean Length Diameter, MLD & 1 & 0 & 0.48 \\
\hline Mean Surface Diameter, MSD & 2 & 0 & 0.59 \\
\hline Mean Volume Diameter, MVD & 3 & 0 & 0.69 \\
\hline Length Mean Diameter, LMD & 2 & 1 & 0.73 \\
\hline Sauter Mean Diameter, SMD & 3 & 2 & 0.88 \\
\hline Volume Mean Diameter, VMD & 4 & 3 & 1.09 \\
\hline
\end{tabular}

Table 1: The variation of different representative diameters for the reference case. $d_{2}$ and $d_{3}$ in equations 3 and 5 - 9 are equivalent to $d_{20}$ and $d_{30}$, respectively in equation 11 .

It is evident from Table 1 that these representative diameters vary greatly and caution should be exercised in using the correct weighted mean diameter for the considered purpose. For instance, the value of SMD is significantly larger than MSD indicating the effect of large bubbles on SMD. However, as pointed out previously, SMD (or $\left.d_{32}\right)$ is the correct representative bubble size for mass transfer models.

The SMD of bubbles in the wake varies with experimental conditions. Trends are observed with the variation of ventilation airflow rate $(Q)$ and cross-sectional mean water velocity $(U)$. Figure 6 shows the effect of variation of these parameters as a function of vertical location in the wake. 
Since the bubble images spanned a finite width in the stream-wise direction $(\sim 60 \mathrm{~mm})$, the bubble data are averaged along the image width to get a variation along the vertical axis. Several observations can be made from this figure.

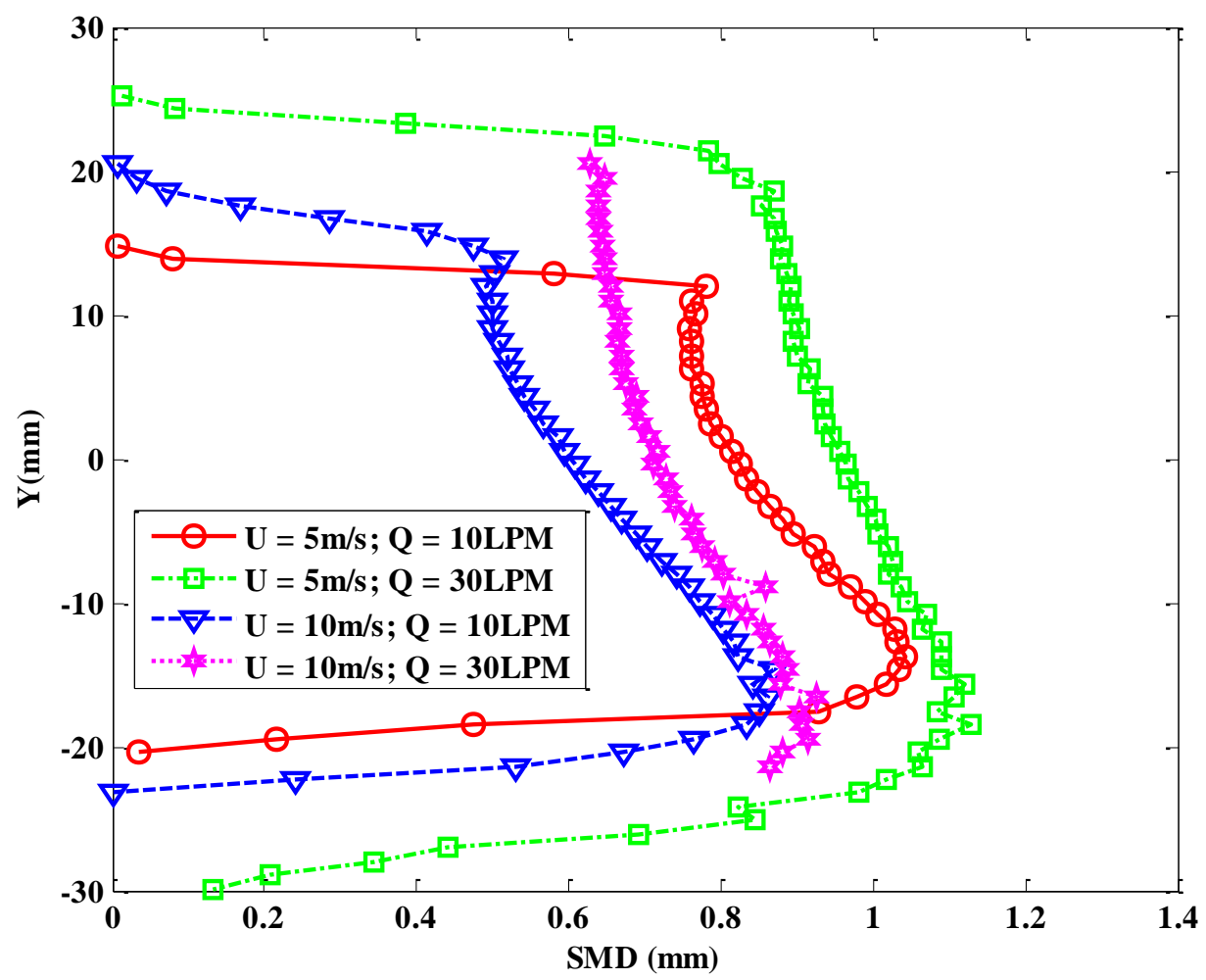

Figure 6: Effect of variation of liquid velocity or ventilation flow on SMD across all the vertical positions in the wake.

The increase in SMD moving from outside toward the center of wake is due to the inertial effects of bubble size. Also, the largest bubbles are found below the centerline due to the ventilation slot being positioned on the suction side, which was located on the bottom of the hydrofoil in the test setup. The rise velocity of bubbles of all sizes is small compared to the liquid velocity [31] but the inertial effect of added or virtual mass is substantial for large bubbles. It is observed that a higher liquid velocity led to a lower SMD (compare red circles with blue triangles). This could be due to the influence of liquid velocity on the production of bubbles [20] or the greater turbulence at higher liquid velocities leading to breakup of bubbles into smaller sizes [30]. At a 
fixed liquid velocity, an increase in ventilation flow led to an increase in SMD (compare red circles with green squares). The increase in SMD is likely due to the influence of ventilation flow rate on bubble diameter [20].

\begin{tabular}{|l|l|l|l|l|}
\hline $\begin{array}{l}\text { AoA }^{+} \\
(\mathrm{deg})\end{array}$ & $\begin{array}{l}U \\
(\mathrm{~m} / \mathrm{s})\end{array}$ & $\begin{array}{l}Q \\
(\text { SLPM })\end{array}$ & $\begin{array}{l}\hat{U}_{32} \\
(\mathrm{~mm})\end{array}$ & $\beta$ \\
\hline 8 & 5 & 10 & 0.79 & 0.54 \\
8 & 5 & 30 & 0.89 & 0.54 \\
8 & 7.5 & 20 & 0.68 & 0.54 \\
8 & 10 & 10 & 0.51 & 0.34 \\
8 & 10 & 30 & 0.58 & 0.52 \\
\hline 4 & 5 & 10 & 0.84 & 0.54 \\
4 & 5 & 30 & 0.95 & 0.59 \\
4 & 7.5 & 20 & 0.75 & 0.59 \\
\hline 4 & 10 & 10 & 0.58 & 0.59 \\
4 & 10 & 30 & 0.66 & 0.54 \\
\hline 0 & 5 & 10 & 0.88 & 0.60 \\
0 & 5 & 30 & 0.98 & 0.60 \\
0 & 7.5 & 20 & 0.80 & 0.60 \\
0 & 10 & 10 & 0.66 & 0.60 \\
0 & 10 & 30 & 0.75 & 0.60 \\
\hline
\end{tabular}

Table 2 : Description of gas transfer experiments conducted in the present study with all the relevant experimental conditions. AoA is the angle of attack. $d_{32}$ is obtained from images. The $\beta$ values are determined from a fit of the model to experiments.

Table 2 shows the variation of $d_{32}$ across all conditions. A tripling of ventilation flow rate produces an approximately $14 \%$ increase in $d_{32}$ at both water velocities and three angles of attack. Similarly, between a 44 and $55 \%$ increase in $d_{32}$ is observed when water velocity is halved at both airflow rates, and $4^{\circ}$ and $8^{\circ}$ angles of attack. The increase is somewhat smaller (31 and 33\%) at zero angle of attack. It can also be seen from Table 2 that there is a slight decrease in $d_{32}$ with increasing angles of attack, and a consistent trend can be observed.

Figure 7 shows the effect of variation of AoA at a fixed water velocity of $10 \mathrm{~m} / \mathrm{s}$ and ventilation flow rate of 10 SLPM. The maximum of the curve at zero angle of attack is below the centerline. This is again because of the ventilation slot being located on the suction (bottom) side of the 
hydrofoil. As AoA is increased, this maximum shifts upwards following the shift of the entire bubbly wake. Figure 7 indicates that the number of bubbles increases substantially with AoA. The area under these curves would yield the total number of bubbles at each angle of attack. Continuity dictates that for the same ventilation flow rate, an increase in the in number of bubbles with AoA must occur concomitantly with smaller bubble sizes, which is seen in Table 2 . The smaller bubbles, in turn, may be attributed to the increased shearing of bubbles from the injection point and the greater breakdown of bubbles in a more turbulent wake.

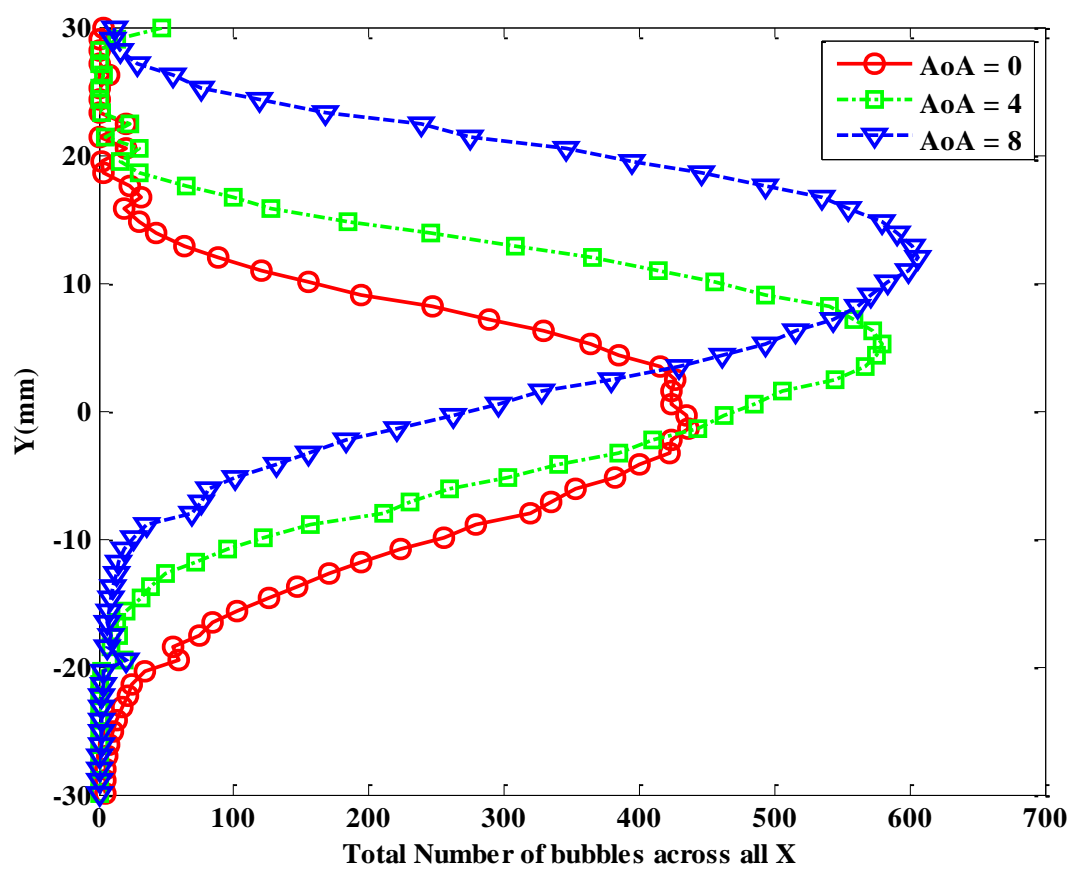

Figure 7: Effect of variation angle of attack (AoA) of hydrofoil on number of bubbles in the wake.

\subsection{Results on Dissolved Oxygen Uptake}

Historically, the $\beta$ coefficient has been difficult to determine, primarily due to the unavailability of data on bubble surface area and the resulting bulk coefficients needed in the analysis $[12,16$, $21,32-36]$. Table 2 shows the variation of the $\beta$ coefficient over the range of experimental conditions tested. It is seen that, except for the $\beta$ value of 0.40 at AoA $=8^{\circ}, \mathrm{V}=10 \mathrm{~m} / \mathrm{s}$, air flow $=10$ SLPM which is considered an outlier, the value of $\beta$ depends only on the hydrofoil 
configuration and has close to a constant value for all the flow conditions at a particular AoA. The $\beta$ coefficient increases from approximately 0.53 to 0.60 as AoA is varied from 0 to $8^{\circ}$. Figure 8 shows percentage saturation $\left(100 C / C_{S}\right)$ over time and the model fit at two air flow rates for an AoA of 0 degress and water velocity of $5 \mathrm{~m} / \mathrm{s}$. The lines represent the experimental data while the marker symbols denote the model best fit using a $\beta$ value of 0.60 . The fact that $\beta$ is less than its expected value (on the order of one) could be due to the cross-sectional mean velocity being substituted for the characteristic turbulent velocity in equation 10 . If the characteristic turbulent velocity is approximately $0.43\left(\mathrm{AoA}=8^{\circ}\right)$ to $0.51\left(\mathrm{AoA}=0^{\circ}\right)$ times the free stream mean velocity, the value of $\beta$, as calculated from equation 10 , would be 1 . A value of 0.43 to 0.51 for the ratio of the characteristic turbulent velocity to mean velocity is realistic for the flow in the wake of a hydrofoil. Determining a $\beta$ value in the range of $0.53-0.60$ for different hydrofoil angles of attack, independent of water velocity and airflow rate, is a seen as a confirmation of Azbel's [28] theory. 


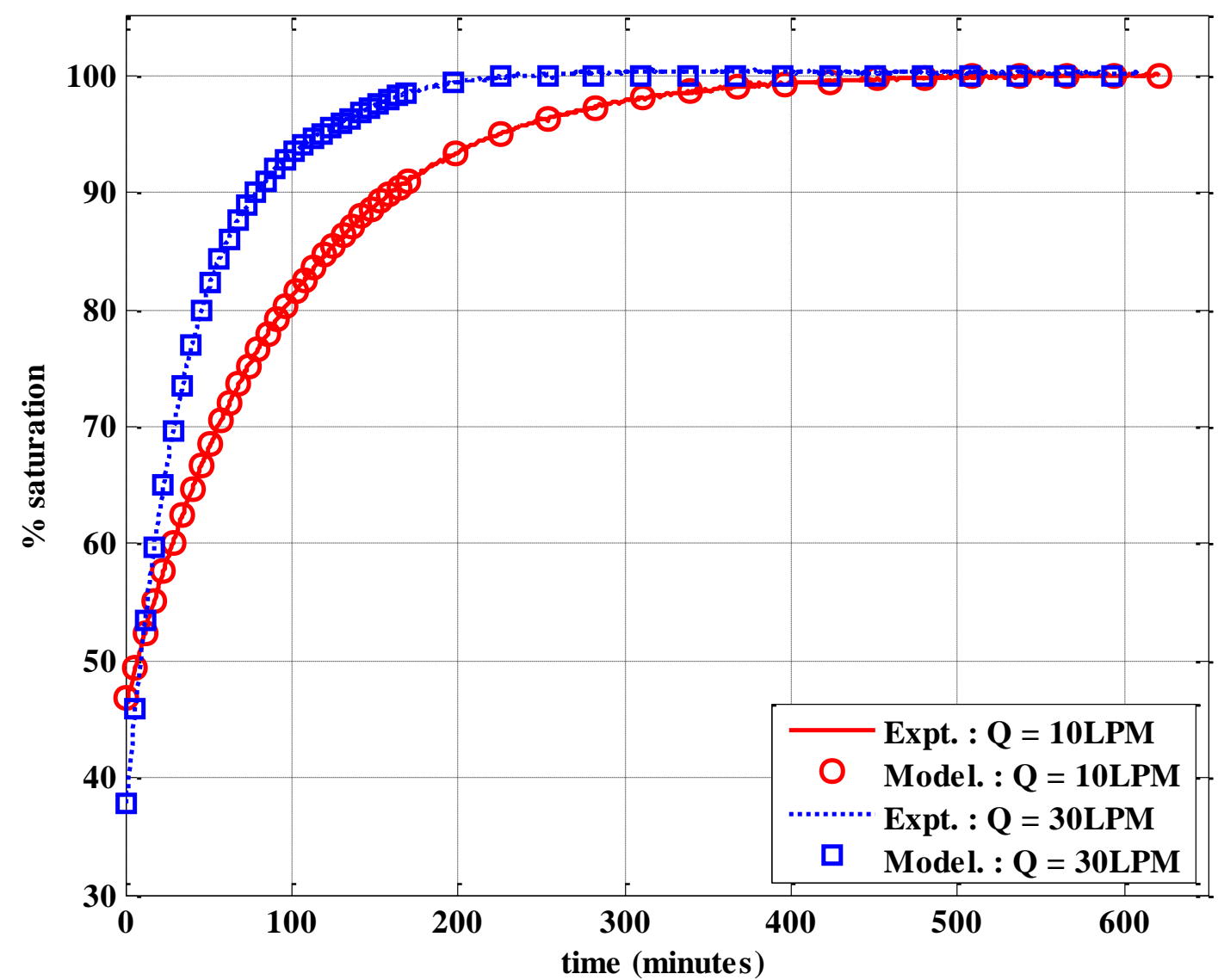

Figure 8: Effect of variation of ventilation rate on \% saturation at zero AoA and superficial liquid velocity, $\mathrm{U}=5 \mathrm{~m} / \mathrm{s}$

\section{Conclusions}

Experiments on bubble-water mass transfer were performed in a closed, recirculating high-speed water tunnel using a ventilated hydrofoil by varying the free stream water velocity, ventilation air flow rate and the hydrofoil angle of attack. The goal of these experiments is to investigate the effect of these parameters on the size of the bubbles generated by air injection and the resulting rate of oxygen transfer. The shadow imaging experiments in the bubbly wake revealed that for our reference case of $0^{\circ} \mathrm{AoA}$, a water velocity of $5 \mathrm{~m} / \mathrm{s}$ and a ventilation mass airflow rate of 10 SLPM, $\sim 40 \%$ of the bubbles in the wake had diameters around $0.3 \mathrm{~mm}$. It is also found that bubbles larger than $0.45 \mathrm{~mm}$ in diameter have a greater influence on the Sauter mean diameter of 
the population. Sauter mean diameter increases by approximately $14 \%$ as a result of tripling the airflow rate at all water velocities. Sauter mean diameter decreased by $44 \%$ to $55 \%$, when the velocity is doubled without changing airflow rate at $4^{\circ}$ and $8^{\circ}$ angle of attacks and by $31 \%$ to 33 $\%$ at zero angle of attack. An increase in angle of attack from 0 to 8 degrees produced smaller bubbles.

A one-dimensional mass transfer model is developed to lend insight into the oxygen transfer characteristics observed in these experiments using a mass transfer conservation equation and a predictive relation for liquid film coefficient, $k_{\mathrm{L}}$, by Azbel [28]. For ease of application, mean free stream velocity $(U)$ was substituted for characteristic turbulent velocity $\left(u^{\prime}\right)$ in the predictive relation. Thus, a $\beta$ coefficient was introduced to account for this difference, $\beta=\left(u^{\prime} / U\right)^{3 / 4}$ where $u^{\prime} / U$ is the turbulence intensity. This $\beta$ coefficient was determined by fitting the prediction of the numerical model with the experimental data obtained from the DO measurements in the watertunnel. The gas transfer model required the test conditions and Sauter mean diameter of the bubbles in the wake of the hydrofoil as input to predict liquid film coefficient, $k_{\mathrm{L}}$. Except for one outlier, the results of the model showed that $\beta$ coefficient is constant for a given angle of attack. $\beta$ decreases slightly as hydrofoil AoA increases. We believe the values of $\beta$ derived in this study can be used with Azbel's $k_{\mathrm{L}}$ relationship as a predictive tool for mass transfer in bubbly flows, which are similar to those studied here. Prior attempts at this have largely been unsuccessful due to the lack of experimental measurements of bubble size distributions, forcing a reliance on empirical formulations for bubble size and surface area.

This study is part of a larger effort to develop computational tools to relate the conditions in a high-speed bubbly flow to the associated gas transfer. The study provides a rich dataset on bubble sizes and void fraction in the wake of a ventilated hydrofoil and the rate of oxygen 
transfer as a function of flow and foil parameters that can be used in the validation of these tools. It is hoped that such computational models will soon be integral parts of the design process in the development of effective auto-venting hydroturbines.

\section{Acknowledgments}

This work was sponsored by the Department of Energy Contract \# DE-EE0005416. Recognition must be given to Mr. Ellison Kawakami currently of 3M Corporation and Dr. Seung-Jae Lee of the Research Institute of Marine Systems Engineering Seoul National University, Korea for their contributions to the initial development of the shadow imaging system.

\section{References}


[1] P. A. March, T. A. Brice, M. H. Mobley, J. M. Cybularz, Turbines for Solving the DO Dilemma, Hydro Review, 11 (1992).

[2] J. S. Gulliver, R. E. A. Arndt, Interfacial transport in river-reservoir systems, Measurement and Modeling of Environmental Flows, American Society of Mechanical Engineers, FED-143 (1992) 77-86.

[3] J. S. Gulliver, Mass transfer in an aerated flows, Second International Symposium on Aeration Technology, American Society of Mechanical Engineers, Washington, D.C., (1998).

[4] E. I. Daniil, J. S. Gulliver, J. R. Thene, Water Quality Impact Assessment for Hydropower, J. Env. Engg., 117(2) (1991) 179-193.

[5] H. Cong, T. Huang, B. Chai, Water-Circulating Aerator: Optimizing Structure and Predicting Water Flow Rate and Oxygen Transfer, J. Hydraul. Eng., 137 (2011) 659-667.

[6] S. O'Boyle, G. McDermott, R. Wilkes, Dissolved oxygen levels in estuarine and coastal waters around Ireland, Mar. Pollut. Bull. 58(11) (2009) 1657-1663.

[7] P. T. Weiss, J. S. Gulliver, M. J. Semmens, "Hypolimnetic Aeration with Hollow Fiber Membranes," Journal of Lake and Reservoir Management, 12(4) (1996) 468-476.

[8] G. C. Buscaglia, F. A. Bombardelli, M. H. Garcia, Numerical modeling of large-scale bubble plumes accounting for mass transfer effects, Int. J. Multiphase Flow 28(11) (2002) 1763-1785.

[9] D.F. McGinnis, A. Lorke, A. Wüest, A. Stöckli, J. C. Little, Interaction between a bubble plume and the near field in a stratified lake, Water Resour. Res. 40(10) (2004) W10206.

[10] E. Alp, C. S. Melching, Allocation of supplementary aeration stations in the Chicago Waterway System for dissolved oxygen improvement, J. Environ. Manage. 92(6) (2011) 1577-1583.

[11] D. E. Hibbs, J. S. Gulliver, Prediction of an effective saturation concentration at spillway plunge pools, J. Hydraul. Eng. 123(11) (1997) 940-949.

[12] A. L. Urban, J. S. Gulliver, D. W. Johnson, Modeling total dissolved gas concentration downstream of spillways, J. Hydraul. Eng. 134(5) (2008) 550-561.

[13] K. P. Parkhill, J. S. Gulliver, Indirect Measurement of Oxygen Solubility, Water Res. 31(10) (1997) 2564-2572.

[14] D. C. Raney, Turbine aspiration for oxygen supplementation, J. Envir. Engrg. Div., ASCE, 103(2) (1977) 341 352.

[15] D. C. Raney, T. G. Arnold , Dissolved oxygen improvement by hydroelectric turbine aspiration, J. Power Div., ASCE, 99(1) (1973) 139-154.

[16] E. J. Thompson, J. S. Gulliver, Oxygen Transfer Similitude for Vented Hydroturbine, J. Hydraul. Eng. 123(6) (1997) 528-538.

[17] R. E. A. Arndt, V. H. Arakeri, H. Higuchi, Some observations of tip-vortex cavitation, J. Fluid Mech. 229 (1991) 269-289.

[18] M. Kjeldsen, R. E. A. Arndt, M. Effertz, Spectral characteristics of sheet/cloud cavitation, J. Fluids Eng. 122(3) (2000) 481-487.

[19] G. M. Monson., Study of Mass Transfer across hydrofoils for use in aerating turbines, Master's thesis. Saint Anthony Falls Laboratory, University of Minnesota (2013). 
[20] E. Silberman, Production of bubbles by the disintegration of gas jets in liquid, Proc. 5th Midwestern Conference on Fluid Mechanics, U of Mich., (1957)

[21] J. P. Giovannettone, J. S. Gulliver, Gas Transfer and Liquid Dispersion Inside a Deep Airlift Reactor, AIChE J. 4(54) (2008) 850-861.

[22] L. P. Goss, J. Estevadeoral, J. W. Crafton, Velocity Measurements Near Walls, Cavities, and Model Surfaces Using Particle Shadow Velocimetry (PSV), 22nd International Congress on Instrumentation in Aerospace Simulation Facilities (2007).

[23] D. Bröder, M. Sommerfeld, Planar shadow image velocimetry for the analysis of the hydrodynamics in bubbly flows, Meas. Sci. Technol. 18 (2007) 2513-2528.

[24] A. Karn, C. Ellis, R. Arndt, J. Hong, An integrative image measurement technique for dense bubbly flows with a wide size distribution, Chem. Eng. Sci. 122 (2015) 240-249.

[25] F. Meyer, Topographic distance and watershed lines. Signal Processing, 38(1) (1994) 113-125.

[26] J. Sauter, Determining size of drops in fuel mixture of internal combustion engines, National Advisory Committee for Aeronautics, (1926).

[27] E. I. Daniil, J. S. Gulliver, Temperature dependence of liquid-film coefficient for gas transfer, J. Environ. Eng. 114(5) (1988) 1224-1229.

[28] D. Azbel, Two-phase flows in chemical engineering, K. Philip, Ed., New York, New York: Cambridge, (1981) 195.

[30] Y. Liao, D. Lucas, A literature review of theoretical models for drop and bubble breakup in turbulent dispersions, Chem. Eng. Sci. 64 (2009) 3389-3406.

[31] W. L. Haberman, R. K. Morton, An experimental investigation of the drag and shape of air bubbles rising in various liquids. Rep. No., 802, NS 715-102, Navy Dept., David Taylor Model Basin, Gaithersburg, MD. 1953.

[32] D. A. Geldert, J. S. Gulliver, S. C. Wilhelms, Modeling dissolved gas supersaturation below spillway plunge pools, J. Hydraul. Eng. 124(5) (1998) 513-521.

[33] C. C. DeMoyer, E. Schierholz, J.S. Gulliver, S.C. Wilhelms, Impact of bubble and free surface oxygen transfer on diffused aeration systems, Water Res. 37 (2003) 1890-1904.

[34] S. C. Wilhelms, J. S. Gulliver, Gas transfer, cavitation and bulking in self-aerated spillway flow," J. Hydraul. Res. 43(5) (2005) 532-539.

[35] E. L. Schierholz, J. S. Gulliver, S.C. Wilhelms, H.E. Henneman, Gas transfer from air diffusers, Water Res. 40(5) (2006) 1018-1026.

[36] A. M. Witt, J. S. Gulliver, Predicting oxygen transfer efficiency at low-head gated sill structures, J. Hydraul. Res. 50(5) (2012) 521-531. 